\title{
Use of dietary supplements in Denmark is associated with health and former smoking
}

\author{
Vibeke K Knudsen 1,2,3, * Lone B Rasmussen ${ }^{1}$, Jóhanna Haraldsdóttir ${ }^{2}$, Lars Ovesen ${ }^{\text {, }}$ \\ Inge Bülow ${ }^{4}$, Nils Knudsen ${ }^{5}$, Torben Jørgensen ${ }^{6}$, Peter Laurberg ${ }^{4}$ and Hans Perrild ${ }^{5}$ \\ ${ }^{1}$ Institute of Food Research and Nutrition, Danish Veterinary and Food Administration, Søborg, Denmark: ${ }^{2}$ Research \\ Department of Human Nutrition, Royal Veterinary and Agricultural University, Copenhagen, Denmark: ${ }^{3}$ Present \\ address: Danish Epidemiology Science Centre, Statens Serum Institute, Artillerivej 5, DK-2300 Copenhagen S, \\ Denmark: ${ }^{4}$ Department of Endocrinology and Medicine, Aalborg Hospital, Aalborg, Denmark: ${ }^{5}$ Department of \\ Internal Medicine I, Bispebjerg Hospital, Copenhagen, Denmark: ${ }^{6}$ Centre for Preventive Medicine, Glostrup \\ Hospital, Glostrup, Denmark (DANTHYR)
}

Submitted 9 July 2001: Accepted 28 September 2001

\begin{abstract}
Objectives: To describe the use of dietary supplements in a group of Danish adults and to investigate the differences between users and non-users with respect to age, gender, health and lifestyle factors.

Design: Cross-sectional study in two Danish cities.

Setting: The Danish Investigation on Iodine Intake and Thyroid Diseases, 1997-1998.

Subjects: Participants were 3707 women (selected age groups between 18 and 65 years) and 942 men (60-65 years). Participation rate was 50\%. Supplement data were collected in a personal interview. Data on education, smoking, alcohol intake, physical activity, use of medication and self-perceived health were derived from a self-administered questionnaire. Multiple logistic regression was used for the statistical analysis.

Results: Participants were asked about all kinds of supplements, ranging from products containing vitamins and minerals, to fish oils and products of herbal origin. On average $59 \%$ reported use of some kind of dietary supplement. Most common were combined multivitamin/mineral products (48\%) followed by single vitamin C products (10\%). Twelve per cent used three or more different supplements. Supplement use was strongly associated with age and gender, being highest among elderly women (78\%). Ex-smokers were more likely to use supplements than subjects who had never smoked (odds ratio (OR) 1.36, 95\% confidence interval (CI) 1.091.76). Supplement use was more likely among subjects who had many days of illness $(\mathrm{OR}=1.37,95 \% \mathrm{CI} 1.12-1.66)$ and among users of medication $(\mathrm{OR}=1.22,95 \% \mathrm{CI}$ 1.04-1.42). Subjects who perceived their health as poor were more likely to use supplements other than just a multivitamin/mineral tablet $(\mathrm{OR}=2.2,95 \%$ CI 1.31 3.77).

Conclusions: Use of dietary supplements was related to age, gender and smoking, but also to poor self-perceived health status and absence from work. This indicates that a group of people use supplements as a form of self-medication.
\end{abstract}

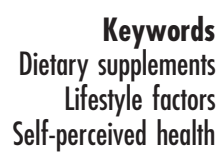

Use of dietary supplements is common in Denmark. According to a national dietary survey in 1985, 60\% of women and $50 \%$ of men (15-80 years) used vitamin $/ \mathrm{min}$ eral supplements ${ }^{1}$. A survey on elderly people in 12 European countries in 1989 showed that the elderly in Denmark had the highest intake of dietary supplements, with $40-50 \%$ of the elderly taking supplements ${ }^{2}$. This is despite the fact that healthy people are not recommended to take dietary supplements by any authority.

Earlier studies have indicated that use of dietary supplements is associated with lifestyle factors, and that supplement users have a healthier lifestyle than non-users ${ }^{3}$. Users have been found to be better educated, less likely to smoke, to drink less alcohol and to exercise more than nonusers $^{4,5}$. In Denmark little is known about how such factors are associated with the use of dietary supplements.

The aim of the present study was to describe the use of dietary supplements and to investigate the differences between users and non-users with respect to age, gender, health and selected lifestyle factors. 


\section{Subjects and methods}

\section{Study population}

The data used in this survey are derived from The Danish Investigation of Iodine Intake and Thyroid Diseases ${ }^{6}$, which was carried out from March 1997 to June 1998. The participants were randomly selected from two cities in Denmark, Aalborg and Copenhagen. A sample was drawn from the civil registration system of all inhabitants in the two cities comprising the following groups: women aged $18-22,25-30,40-45$ and 60-65 years, and men aged 6065 years. Sampling was stratified in order to obtain equal numbers in each of the groups. An overrepresentation of young subjects was selected as the study was designed for follow-up. Since thyroid diseases are much more widespread in women than men, the participants included in the survey comprised more women than men, with the women in the survey representing a wider age range than the men. Men in the age group 60-65 years were included to allow comparisons between genders. This age group was chosen because the number of cases with thyroid abnormalities is supposed to increase with age. Of the 9274 persons invited, 4649 (50\%) participated in the survey. Participation rates in the groups were $46.3 \%$, $55.3 \%, 62.5 \%, 42.8 \%$ and $47.0 \%$, and $49.9 \%$, respectively. The sample has been described in detail elsewhere ${ }^{6}$.

\section{Data collection}

All participants received a mailed questionnaire about smoking habits, alcohol intake, physical activity, education and employment, medical history, days absent from work due to illness, and they were asked about their selfperceived health status. Participants were invited to a medical examination, which included measurements of weight and height and a personal interview on use of medications, dietary supplements, tobacco and alcohol.

In the questionnaire the participants were asked if they used any kind of dietary supplements (including herbal products) and medication, and they were asked to bring all products to the medical examination and interview. The interviewer, a medical doctor, asked for detailed information, and noted brand name, dose and period of use.

\section{Dietary supplements}

Before data analysis, the dietary supplements were grouped according to their type and content of nutrients into the following groups: (1) multivitamin and/or mineral products; (2) vitamin $\mathrm{C}$ products, which included all products with vitamin C only; (3) vitamin B products, which included single vitamin $\mathrm{B}$ and complexes of vitamin B; (4) calcium products, which also included products with calcium and vitamin D; (5) iron products, which included products with iron only as well as iron products with and without vitamin C; (6) a group of other products with single minerals or vitamins; (7) garlic preparations;
(8) fish oils (including cod liver oil); (9) ubiquinoncontaining products $\left(\mathrm{Q}_{10}\right)$; and (10) a group of 'Other dietary supplements' including e.g. ginseng products, fibre products and other herbal products. Supplements were either in tablet or capsule form, or as powder or fluid.

\section{Users of supplements}

A user of dietary supplements was defined as a person who took one or more of the supplements mentioned above at the time of the survey. Furthermore, we defined a subgroup of users comprising all of the participants who took a multivitamin/mineral supplement and nothing else.

\section{Statistical analysis}

Associations between lifestyle and health factors and use of dietary supplements, as well as use of multivitamin/ mineral supplements only, were analysed by multiple logistic regression. The following factors were tested in the multiple logistic regression models: age, body mass index, physical activity, education, geographic region (Aalborg vs. Copenhagen), smoking habits (smokers, non-smokers (who have never smoked), occasional smokers and former smokers), alcohol intake (total number of drinks per week, and separately for beer, wine and spirits), use of drugs (use/no use at the time of the survey), contact with health authorities during the last year, days absent from work due to illness during the past year, tiredness (yes or no to the question: 'Do you feel tired at the moment?') and self-perceived health status rated on a scale from 1 (very good) to 5 (very poor), which was simplified to three groups in the analysis, by adding the two first and the two last categories. The variables that were significantly associated with supplement use $(P<0.05)$ were kept in the model while the variables with no significant association $(P>0.05)$ were left out (stepwise backward regression). Because of the very skewed distribution of age and gender, only women were included in these analyses. For comparison of supplement use among men and women (60-65 years), the chi-square test was used. All analyses were carried out by use of SPSS for Windows (SPSS Inc., Chicago, IL).

\section{Results}

\section{Use of supplements}

Of the 4649 subjects who participated in the survey, 2758 (59\%) reported use of dietary supplements (Table 1). Multiple vitamin/mineral tablets were most common, used by $48 \%$. Among the single vitamin or mineral products, vitamin C, vitamin B (either single supplements or complexes) and calcium were most common. Fish oil was used by approximately $7 \%$ of the participants (Table 1). Thirteen per cent used 'Other supplements', a large and heterogeneous group comprising a large variety of specified products as well as unspecified products.

Some $(27 \%)$ of the participants took more than one 
Table 1 Use of different dietary supplements. Number (\%) of participants

\begin{tabular}{|c|c|c|c|c|c|c|c|}
\hline \multirow[b]{2}{*}{ Age (years) } & \multicolumn{4}{|c|}{ Women } & \multirow{2}{*}{$\frac{\text { Men }}{60-65}$} & \multirow[t]{2}{*}{$P \|$} & \multirow[t]{2}{*}{ Total } \\
\hline & $18-22$ & $25-30$ & $40-45$ & $60-65$ & & & \\
\hline$n$ & 957 & 943 & 918 & 889 & 942 & $<0.001$ & 4649 \\
\hline Any kind & $445(46.5)$ & $517(54.8)$ & $540(58.8)$ & 694 (78.1) & $562(59.7)$ & $<0.001$ & 2758 (59.3) \\
\hline Multivitamins* & $390(40.8)$ & $462(49.0)$ & 446 (48.6) & $533(60.0)$ & 395 (41.9) & $<0.001$ & 2228 (47.9) \\
\hline Vitamin C† & $61(6.4)$ & $72(7.6)$ & $80(8.7)$ & $150(16.9)$ & $84(8.9)$ & $<0.001$ & 447 (9.6) \\
\hline Vitamin B† & 40 (4.2) & $54(5.7)$ & $47(5.1)$ & $93(10.4)$ & $52(5.5)$ & $<0.001$ & $286(6.1)$ \\
\hline Calcium & $23(2.4)$ & $33(3.5)$ & $61(6.6)$ & $120(13.5)$ & $10(1.1)$ & $<0.001$ & 247 (5.3) \\
\hline Iron & 32 (3.3) & 67 (7.1) & 35 (3.8) & $13(1.5)$ & 11 (1.2) & 0.682 & $158(3.4)$ \\
\hline Minerals $\ddagger$ & $24(2.5)$ & $24(2.6)$ & $24(2.6)$ & 30 (3.3) & $7(0.7)$ & $<0.001$ & 109 (2.3) \\
\hline Garlic products & $10(0.1)$ & $7(0.7)$ & $23(2.5)$ & $84(9.4)$ & $58(6.2)$ & 0.009 & $182(3.9)$ \\
\hline Fish oils & $11(1.1)$ & $11(1.2)$ & $37(4.0)$ & 163 (18.3) & $132(14.0)$ & 0.013 & 354 (7.6) \\
\hline Ubiquinon, $\mathrm{Q}_{10}$ & $4(0.4)$ & $12(1.3)$ & $22(2.4)$ & $46(5.2)$ & $44(4.7)$ & 0.666 & $128(2.8)$ \\
\hline Others§ & $81(8.4)$ & $96(10.1)$ & $110(12.0)$ & 218 (24.5) & $98(10.4)$ & $<0.001$ & 603 (13.0) \\
\hline
\end{tabular}

* Multivitamin and/or multimineral supplements.

† Single vitamins.

$\ddagger$ Single minerals, e.g. selenium and zinc.

$\S$ Other kinds of dietary supplement, i.e. fibre products, gingko biloba and unspecified supplements.

q $P$-values for comparison of men and women age 60-65 years, chi-square test

dietary supplement per day, $12 \%$ took three or more (Table 2). Supplement use was most common among the women in the age group 60-65 years, among whom $78 \%$ were supplement users and $26 \%$ used three or more dietary supplements per day (Table 2).

\section{Supplement users}

Female supplement users were compared with female non-users with respect to lifestyle and health factors in a multiple logistic regression model. Age and supplement use were strongly associated, with the youngest women, 18-22 years, being the least likely users (Table 3). Educational status was related to supplement use although no linear association was found. There were more users among women with short and middle-length education, and students, compared with women with primary school only. Also the group of women with academic education tended to have more users, but this trend was not statistically significant (Table 3). Supplement use tended to be less common among obese women (body mass index (BMI) $>30 \mathrm{~kg} \mathrm{~m}^{-2}$ ) compared with normal weight women $\left(B M I=20-25 \mathrm{~kg} \mathrm{~m}^{-2}\right)$ (Table 3). Furthermore, smoking habits were associated with supplement use, with exsmokers being significantly more likely to use supplements than subjects who had never smoked and daily smokers (Table 3). While total alcohol intake showed no relation to supplement use there was a negative association with beer intake, and women who consumed more than 13 beers per week were significantly less likely to be users (Table 3). Absence from work due to illness was associated with supplement use, with more users among those who had 7 or more days' absence during the last year (Table 3). In addition there were more supplement users among women who reported use of medication and among women who felt tired (Table 3).

The next step in the analysis was to compare two groups of dietary supplement users: one group who only used multivitamin/mineral tablets and another group who used other types of supplement (alone or in addition to multivitamin/mineral tablets). This comparison showed an association with age and self-perceived health. The probability of being a multivitamin/mineral user only decreased with increasing age and with increasingly poor self-perceived health (Table 4). This means that older women, and women who perceived their health as 'poor' or 'very poor', were more likely to use supplements other than just a multivitamin/mineral tablet.

\section{Men versus women}

Comparison of men and women was only possible for the age group 60-65 years due to the composition of the sample (see Subjects and methods). Within this age group fewer men than women took any kind of dietary supplement ( $60 \%$ vs. $78 \%, P<0.001$, Table 1). This was valid for all kinds of supplement, except iron and ubiquinon supplements (Table 1). Furthermore, only

Table 2 Number of different supplements used by participants (percentage of participants)

\begin{tabular}{|c|c|c|c|c|c|c|}
\hline \multirow[b]{2}{*}{ Age (years) } & \multicolumn{4}{|c|}{ Women } & \multirow{2}{*}{$\frac{\text { Men }}{60-65}$} & \multirow[t]{2}{*}{ Total } \\
\hline & $18-22$ & $25-30$ & $40-45$ & $60-65$ & & \\
\hline$n$ & 957 & 943 & 918 & 889 & 942 & 4649 \\
\hline No supplements & 512 (53.3) & $426(45.2)$ & 378 (41.2) & 195 (21.9) & $380(40.3)$ & $1891(40.7)$ \\
\hline One supplement & 279 (29.2) & 321 (34.0) & $310(33.8)$ & $270(30.4)$ & $331(35.1)$ & 1511 (32.5) \\
\hline Two supplements & $107(11.2)$ & $113(12.0)$ & $132(14.4)$ & 197 (22.2) & $143(15.2)$ & $692(14.9)$ \\
\hline Three supplements or more & $59(6.2)$ & $83(8.8)$ & $98(19.7)$ & $227(25.5)$ & $88(9.3)$ & $555(11.9)$ \\
\hline
\end{tabular}


Table 3 Logistic regression analyses of associations between use of dietary supplements and various background variables. Odds ratio for using supplements. Subjects were classified as supplement users $(n=1849)$ or non-users $(n=1339)$

\begin{tabular}{|c|c|c|c|c|}
\hline & $n$ & OR & $95 \% \mathrm{Cl}$ & $P$ \\
\hline Age group* & & & & $<0.001$ \\
\hline $18-22$ years & 863 & 1.00 & & \\
\hline $25-30$ years & 832 & 1.40 & $1.12-1.75$ & 0.004 \\
\hline $40-45$ years & 809 & 1.85 & $1.44-2.38$ & $<0.001$ \\
\hline $60-65$ years & 684 & 5.50 & $4.11-7.37$ & $<0.001$ \\
\hline Education & & & & 0.019 \\
\hline Primary school only & 578 & 1.00 & & \\
\hline Student/trainee & 600 & 1.50 & $1.15-1.94$ & 0.002 \\
\hline Semi-skilled worker & 65 & 1.33 & $0.73-2.43$ & 0.359 \\
\hline Short education ( $<1$ year) & 179 & 1.10 & $0.76-1.58$ & 0.617 \\
\hline Craftsman & 415 & 1.18 & $0.89-1.55$ & 0.244 \\
\hline Short theoretic ( $1-2$ years) & 413 & 1.61 & $1.21-2.13$ & 0.001 \\
\hline Medium ( $3-4$ years) & 702 & 1.39 & $1.08-1.79$ & 0.010 \\
\hline Academic (5 years or more) & 236 & 1.37 & $0.98-1.92$ & 0.069 \\
\hline Body mass index $\left(\mathrm{kg} \mathrm{m}^{-2}\right)$ & & & & 0.082 \\
\hline $20-24.9$ & 1696 & 1.00 & & \\
\hline$<20$ & 373 & 1.14 & $0.91-1.45$ & 0.255 \\
\hline $25-29.9$ & 750 & 0.89 & $0.74-1.08$ & 0.246 \\
\hline$\geq 30$ & 369 & 0.76 & $0.61-0.98$ & 0.034 \\
\hline Smoking habits & & & & 0.002 \\
\hline Non-smoker (never smoked) & 1326 & 1.00 & & \\
\hline Daily smoker & 1122 & 0.90 & $0.75-1.07$ & 0.218 \\
\hline Occasional smoker & 158 & 1.15 & $0.82-1.62$ & 0.421 \\
\hline Former smoker & 582 & 1.36 & $1.09-1.70$ & 0.006 \\
\hline Beers per week & & & & 0.012 \\
\hline 0 & 1463 & 1.00 & & \\
\hline $1-4$ & 1435 & 0.97 & $0.83-1.13$ & 0.700 \\
\hline $5-8$ & 187 & 0.77 & $0.55-1.06$ & 0.111 \\
\hline $9-12$ & 57 & 0.74 & $0.42-1.28$ & 0.277 \\
\hline$\geq 13$ & 46 & 0.35 & $0.18-0.67$ & 0.002 \\
\hline Days absent from work & & & & 0.003 \\
\hline 0 & 1229 & 1.00 & & \\
\hline $1-6$ & 1015 & 1.04 & $0.86-1.26$ & 0.675 \\
\hline$\geq 7$ & 944 & 1.37 & $1.12-1.66$ & 0.002 \\
\hline \multicolumn{5}{|l|}{ Medication use } \\
\hline No & 2450 & 1.00 & & \\
\hline Yes & 738 & 1.39 & $1.12-1.73$ & 0.003 \\
\hline \multicolumn{5}{|l|}{ Tiredness } \\
\hline No & 1627 & 1.00 & & \\
\hline Yes & 1561 & 1.17 & $1.01-1.36$ & 0.043 \\
\hline
\end{tabular}

OR - odds ratio; $95 \% \mathrm{Cl}$ - 95\% confidence interval.

* Only female participants are included in this analysis.

$25 \%$ of the men took more than one supplement, compared with $48 \%$ of the women in this age group (Table 2).

To compare the men and women with respect to background variables, a separate analysis for men in the age group 60-65 years was performed. This test showed that supplement use among men was correlated to number of days of illness and use of medication, with the persons having more days off due to illness and who took more medication having a higher probability of taking dietary supplements (Table 5). Furthermore, educational level was associated with supplement use, although no linear trend appeared. Body mass index was not associated with supplement use among men, and neither was smoking, alcohol consumption or tiredness; all of these factors were associated with supplement use among women.
Table 4 Multiple logistic regression restricted to supplement users only. Users of multivitamin/mineral products only $(n=792)$ vs. users of other supplements $(n=1849)$

\begin{tabular}{lrrrr}
\hline & $n$ & OR & $95 \% \mathrm{Cl}$ & \multicolumn{1}{c}{$P$} \\
\hline Age group* & & & & $<0.001$ \\
18-22 years & 395 & 1.00 & & \\
$25-30$ years & 449 & 1.02 & $0.75-1.40$ & 0.882 \\
40-45 years & 468 & 1.52 & $1.10-2.12$ & 0.012 \\
$\quad 50-65$ years & 537 & 3.31 & $2.37-4.62$ & $<0.001$ \\
Education & & & & 0.014 \\
$\quad$ Primary school only & 306 & 1.00 & & \\
$\quad$ Student/trainee & 302 & 0.92 & $0.64-1.33$ & 0.664 \\
$\quad$ Semi-skilled worker & 47 & 1.88 & $0.85-4.12$ & 0.117 \\
$\quad$ Short education (<1 year) & 108 & 1.36 & $0.83-2.23$ & 0.225 \\
$\quad$ Craftsman & 249 & 0.76 & $0.53-1.09$ & 0.133 \\
$\quad$ Short theoretic (1-2 years) & 268 & 0.68 & $0.48-0.97$ & 0.034 \\
$\quad$ Medium (3-4 years) & 436 & 1.05 & $0.76-1.45$ & 0.777 \\
$\quad$ Academic (5 years or more) & 133 & 1.13 & $1.00-1.85$ & 0.591 \\
Self-perceived health & & & & $<0.001$ \\
$\quad$ Very good or Good & 1252 & 1.00 & & \\
$\quad$ Moderate & 511 & 1.38 & $1.10-1.72$ & 0.005 \\
Poor or Very poor & 86 & 2.22 & $1.31-3.77$ & 0.003 \\
\hline OR & & & & \\
\hline
\end{tabular}

OR - odds ratio; $95 \% \mathrm{Cl}-95 \%$ confidence interval.

* Only female participants are included in this analysis.

\section{Discussion}

The results from this survey showed that use of dietary supplements is common in Denmark and that it increases with age. More elderly women than elderly men used dietary supplements. Furthermore, users of dietary supplements differed from non-users with respect to some factors related to lifestyle and health.

Approximately 59\% of the women in this survey used one or more supplements. This proportion is similar to what was reported from a national dietary survey in 1985 , where $60 \%$ of women (15-80 years) used supplements ${ }^{1}$, and this indicates that not much has changed in the 12 years between the two surveys.

Table 5 Logistic regression analyses of associations between use of dietary supplements and various background variables. Odds ratio for using supplements. Only male participants are included in this analysis

\begin{tabular}{lrrlc}
\hline & $n$ & OR & $95 \% \mathrm{Cl}$ & $P$ \\
\hline Education & & & & 0.012 \\
$\quad$ Primary school only & 120 & 1.00 & & \\
$\quad$ Student/trainee & 3 & 1.09 & $0.10-12.67$ & 0.942 \\
Semi-skilled worker & 87 & 0.62 & $0.35-1.10$ & 0.105 \\
$\quad$ Short education (<1 year) & 13 & 0.69 & $0.21-2.24$ & 0.539 \\
Craftsman & 282 & 0.78 & $0.50-1.22$ & 0.278 \\
$\quad$ Short theoretic (1-2 years) & 76 & 2.63 & $1.30-5.32$ & 0.007 \\
$\quad$ Medium (3-4 years) & 159 & 0.92 & $0.56-1.53$ & 0.733 \\
Academic (5 years or more) & 63 & 1.16 & $0.60-2.25$ & 0.652 \\
Days absent from work & & & & 0.028 \\
$\quad$ & 624 & 1.00 & & \\
1-6 & 81 & 1.60 & $0.94-2.70$ & 0.082 \\
$\geq 7$ & 98 & 1.72 & $1.06-2.79$ & 0.028 \\
Medication & & & & \\
$\quad$ No & 425 & 1.00 & & \\
Yes & 378 & 1.66 & $1.11-2.48$ & 0.014 \\
\hline
\end{tabular}

OR - odds ratio; $95 \% \mathrm{Cl}-95 \%$ confidence interval. 
The women in the present survey did not, however, represent the whole population of women in Denmark as they all came from two large cities (Copenhagen and Aalborg) and the participation rate was only $50 \%$. Comparison between men and women, which was only possible for the age group 60-65 years, showed that more women than men used dietary supplements. The difference, twenty percentage points, was of the same order of magnitude as observed in the 1985 survey $^{1}$.

Surveys from comparable countries have reported various results. In Norway a similar proportion, $58 \%$ of adult women (16-79 years), used vitamin and mineral products $^{7}$ whereas in Sweden only 43\% of women (45-65 years) reported taking dietary supplements, in this latter analysis all kinds of supplements were included ${ }^{8}$. In these countries, like in Denmark, the use increased with age. In the Netherlands only $17 \%$ of the population aged $1-75$ years took dietary supplements 5 . In a survey of British women in the age range of $33-72$ years, $61 \%$ of the participants took dietary supplements ${ }^{3}$, which proportion is very similar to the results found in the present survey. Population studies from the USA have reported results varying from $26 \%$ to $45 \%$ among adult women ${ }^{9,10}$. The large deviations in the results are mainly because of the different definitions of dietary supplements used in the surveys. In some of the surveys, the investigators only counted the supplements containing vitamins and minerals, while others included all kinds of dietary supplements, including herbal products ${ }^{8,9}$. Moreover, some investigators categorised a person as a user only if he or she took the supplement on a regular basis every day, while others included the occasional user in the analyses ${ }^{8,9}$.

The present survey showed that the most widely used dietary supplement was multivitamin/mineral products. The second most common supplement was single vitamin C products, followed by single or multiple vitamin B products. Among the supplements with no added nutrients the most common was fish oil followed by garlic products. Surveys from other countries also showed that the most frequently used supplement was multivitamin and mineral products ${ }^{8,11,12}$. In a Dutch survey, garlic supplements were reported to be one of the most widely used supplements, with $2 \%$ of the female participants reporting use of garlic supplements, and the use increased with age ${ }^{5}$. In comparison, the use of garlic supplements in the present survey was $4 \%$ in total, and in the group of women aged $60-65$ years $9 \%$ took garlic supplements. In Norway the most used supplement after multivitamin/ mineral products was cod liver oil, which was used by $33 \%$ of the female participants (aged 30-59 years) ${ }^{7}$.

The common use of several different supplements, in particular among the elderly women where $26 \%$ used three or more, raises the question of unfortunate combinations and the potential hazard of large doses. Unfortunately the data from the present survey were not suitable for dose calculations, as the available information on brand names, concentrations and doses was insufficient for that purpose. As the more elderly used several dietary supplements, this group was obviously less likely to use only a multivitamin/mineral tablet. The elderly women also had the highest percentage of users, no matter what type of dietary supplement (except iron). The smallest differences between age groups were observed for multivitamin/mineral products. This is in accordance with the results from a Dutch survey, which showed that older people more often took dietary supplements of herbal origin compared with the younger age groups, while individuals in all age categories used multivitamin products 5 .

Educational status and supplement use were associated in the present survey, although the association was not linear. The overall tendency was that use of supplements increased with increasing length of education. Similar observations have been reported from other countries, e.g. Sweden and Norway ${ }^{7,8}$, where significant positive association between use of dietary supplements and length of education was found. This relationship might be explained by the increased concern and awareness of a healthy lifestyle among the classes with higher education, which is shown by choosing to take dietary supplements.

Use of dietary supplements was not only related to age, gender and education but also to several lifestyle factors. Use of multiple logistic regression analysis made it possible to investigate these factors with adjustment for other background variables in the group of women. In the analysis of the relationship with smoking habits we split non-smokers up in two categories, ex-smokers and never smokers. The ex-smokers turned out to be the group most likely to use supplements. The same trend was seen in a British survey with female participants in the age group 33-72 years, as the former smokers were more likely to take dietary supplements than non-smokers (who have never smoked), while smokers took less ${ }^{3}$. This could indicate an increased concern about health and choice of a healthier lifestyle among former smokers.

In this survey no relation between total alcohol consumption and supplement use was found, but beer intake was negatively associated to supplement use. Most other surveys that have investigated the supplement/alcohol relationship analysed the total alcohol consumption only, and the results are diverse $e^{3,13}$. One survey, including men as well as women, analysed intake of different types of alcohol in connection with supplement use, and found a smaller proportion of supplement users among beer drinkers compared with wine drinkers ${ }^{9}$. After controlling for age and socio-economic factors, the difference was no longer significant.

The present observation that obese women (BMI > $30 \mathrm{~kg} \mathrm{~m}^{-2}$ ) were less likely to be supplement users than normal weight women is supported by other studies which reported similar results after controlling for age and 
gender ${ }^{3,13,14}$. This might indicate a greater awareness of health and healthy lifestyle among normal weight and lean women than obese women.

The other health factors included in the present study were self-perceived health, tiredness, use of medication and the number of days absent from work during the last year. All of these factors were associated with supplement use and pointed in the same direction: that those subjects with poorer health were more likely to use dietary supplements. For self-perceived health the association was slightly more subtle than for the other parameters as it was mainly a question of the kind of supplement used; subjects who perceived their health as 'poor' or 'very poor' were more likely to use supplements other than multivitamin/mineral tablets. This relationship between health and supplement use indicates a tendency to use dietary supplements as a self-medication to cope with tiredness and poor health. This is supported by a Danish study on the use of ubiquinon-containing supplements $\left(\mathrm{Q}_{10}\right)$, where subjects were asked why they used the supplement; the most frequent answers were 'tiredness' and 'to improve health ${ }^{15}$. The results from this survey thus underline the relevance of further investigations in the area of self-medication and the reasons for taking dietary supplements.

The present study gave limited opportunities to analyse supplement use in men, as only men in the age group 6065 years were included. Body mass index was associated with supplement use in women but not in men. The trend that supplement users are more ill and use more medication than non-users is not as strong in men as in women, although apparent to some extent.

In conclusion, female users and non-users of dietary supplements differed with respect to certain health and lifestyle factors. This should be taken into account in epidemiological studies relating nutrient intake and disease, as use of supplements otherwise may act as a confounding factor. The results suggest that supplement users, or a subgroup of supplement users, are concerned about their health and may have reason to be so, and that they use dietary supplements to cope with tiredness and poor health.

\section{Acknowledgements}

The Danish Investigation on Iodine Intake and Thyroid Diseases, 1997-1998 was supported by grants from the Tømmerhandeler Vilhelm Bang Foundation, the Copenhagen Hospital Corporation Research Foundation, the 1991 Pharmacy Foundation, the Danish Medical Foundation, the Health Insurance Foundation and North Jutland County Research Foundation.

\section{References}

1 Haraldsdottir J, Holm L, Jensen JH, Møller A. Dietary Habits in Denmark 1985. 2. Who eats what? [in Danish with an English summary]. Publication No. 154:293-94. Copenhagen: National Food Agency, 1987.

2 Cruz JA, Moreiras-Varla O, van Staveren WA, Trichopoulou A, Roszkowski W. Intake of vitamins and minerals. Euronut SENECA investigators. Eur. J. Clin. Nutr. 1991; 45(Suppl. 3): 121S-38S.

3 Kirk SFL, Cade JE, Barrett JH, Conner M. Diet and lifestyle characteristics associated with dietary supplement use in women. Public Health Nutr. 1999; 2: 69-73.

4 Lyle BJ, Mares-Perlman JA, Klein BE, Klein K, Greger JL. Supplement users differ from nonusers in demographic, lifestyle, dietary and health characteristics. J. Nutr. 1998; 128: 2355-62.

5 Dorant E, Brandt PVD, Hamstra AM, Feenstra MH, Goldbohm RA, Hermus RJJ, Sturmans F. The use of vitamins, minerals and other dietary supplements in The Netherlands. Int. J. Vit. Nutr. Res. 1993; 63: 4-10.

6 Knudsen N, Bülow I, Jørgensen T, Laurberg P, Ovesen L, Perrild H. Goitre prevalence and thyroid abnormalities at ultrasonography: a comparative epidemiological study in two regions with slightly different iodine status. Clin. Endocrinol. 2000; 53: 479-85.

7 Johansson L, Solvoll K. Norkost 1993-4 og 1997. In: Landsomfattende kostholdsundersøkelser blant menn og kvinner $i$ alders 16- 79 år [National Dietary Surveys Among Men and Women, 16- 79 years]. Publication No. 3:57. Oslo: Norwegian National Council of Nutrition and Physical Activity, 1999.

8 Wallström P, Elmståhl S, Hanson BS, Östergren P, Johansson U, Janzon L, Larsson SA. Demographic and psychosocial characteristics of middle-aged women and men who use dietary supplements. Results from the Malmö Diet and Cancer Study. Eur. J. Public Health 1996; 6: 1888-95.

9 Block G, Sinha R, Gridley G. Vitamin supplement use by demographic characteristics. Am. J. Epidemiol. 1988; 127: 297-309.

10 Stewart ML, McDonald JT, Levy AS, Schucker RE, Henderson DP. Vitamin/mineral supplement use: a telephone survey of adults in the United States. J. Am. Diet. Assoc. 1985; 85 1585-90.

11 Eldrigde AL, Sheehan ET. Food supplement use and related beliefs: survey of community college students. J. Nutr. Educ. 1994; 26: 259-65.

12 Bazzarre T, Hopkins RG, Wu SL, Murdoch S. Chronic disease risk factors in vitamin/mineral supplement users and nonusers in a farm population. J. Am. Coll. Nutr. 1991; 10: $247-57$

13 Kim I, Williamson DF, Byers T, Koplan J. Vitamin and mineral supplement use and mortality in a US cohort. Am. J. Public Health 1993; 83: 546-50.

14 Read MA, Brunner RL, Miller G, St Jeor ST, Scott BJ, Carmody TC. Relationship of vitamin/mineral supplementation to certain psychological factors. J. Am. Diet. Assoc. 1991; 91 : 1429-31.

15 Overvad K, Diamant B, Holm L, Hølmer G, Mortensen SA, Stender S. Efficacy and safety of dietary supplementation containing Q10 [in Danish]. Ugeskr. Laeger 1997; 159: 7309-15. 